\title{
FIVE-CHANNEL PROGRAMMABLE COUNTER
}

\author{
Michael D. Polito \\ Environmental Measurements Laboratory, U.S. Department of Energy \\ 376 Hudson Street, New York, NY 10014-3621
}

\begin{abstract}
A five-channel programmable counter, constructed in a two-wide nuclear instrument module, is described. This counter is versatile in performance and flexible to changes, yet it is implemented with a minimum of hardware through the use of an onboard computer and a very large scale integrated counting circuit. The instrument is able to simultaneously count pulses at rates up to $7 \mathrm{MHz}$ from five different sources for a period of time specified by the user. It stores data internally and can be used as a stand-alone counter or connected to a computer, modem or printer through a standard RS-232 serial communications port.
\end{abstract}

\section{INTRODUCTION}

Counting applications are fundamental to laboratories working with radionuclides or analyzing radioactive substances. Many laboratory counting systems are bulky and require a great deal of ancillary equipment. Special datetime modules and Teletype control modules are often needed and each counter usually occupies its own nuclear instrument module (NIM)[1]. Such systems are costly to maintain and equipment from one system cannot usually be used on other systems. Furthermore, most of the current counter modules have no internal data storage capability. Data must be outputted to paper or magnetic tape, then transferred to a computer for analysis and storage; often a time consuming and tedious process.

Some earlier counter module designs developed at the Environmental Measurements Laboratory (EML) dealt with many of these problems. SMART-I and Clever Counter multichannel counting systems[2] were compact and troublefree. Their counting parameters, such as setting the active channels, date/time clock, and counting interval, were programmable from a Teletype or computer. A magnetic cassette recorder was used for data storage. However, the systems did not store data internally, had no front panel display and were difficult to modify. Changing their software required assembly language programming on special equipment, followed by erasing and reprogramming a new set of electrically programmable read only memories (EPROMs). Unable to store data internally and without a front panel display, they could not serve as stand-alone instruments.

\section{DESIGN OVERVIEW}

Our goal was to replace these older counting systems with a compact and flexible counter module that would require no special ancillary equipment and that could be accessed directly by a computer or terminal, yet also function as a stand-alone counting system. In order to function as a stand-alone instrument, front panel controls for setting counting parameters and a display for data were needed.

Along with storing data internally in non-volatile random access memory (RAM), other essential requirements were that the instrument have multiple counting channels and the capability of being reprogrammed in a high-level language by the user. This concept makes these programming procedures readily available and thus easily modified by the user.

The unique features of this new design were implemented by combining a single-card computer from Onset Computer Corporation [3] with a very large scale integrated (VLSI) circuit, the Am9513A, System Timing Controller from Advanced Micro Devices Incorporated [4]. An important feature of the on-board computer is that it is programmable in BASIC as well as in assembly language. Advantages of the Am9513A are its multichannel, high counting rate capability and its variety of operating modes. The resulting system is a versatile instrument that is not only easy to use, but because of the design, it is readily adapted by the user to suit his particular experimental needs or preferences.

\section{OPERATION AND APPLICATIONS}

When power is first applied to the instrument, an assembly language program is executed which configures the Am9513A as five separate counters. All counting parameters from the previous use--including stored data and user-defined counting times--have been preserved. A BASIC program then controls the instrument's operation. The user interacts with this program via either push buttons on the front panel or a menu shown on an external computer or terminal. Fig. 1 shows a front panel view. The push buttons are simple to operate, much like the controls on a video cassette recorder (VCR). One button selects a 
function, while the other allows the user to set it. Via the push buttons or computer the user can:
a) set the counting interval,
b) set or read the date/time clock,
c) download the data file,
d) clear the data file,
e) monitor intermediate results,
f) change baud rates,
g) exit the program

and several other functions; e.g., selecting which channels will be displayed on the front panel and whether to continue a counting cycle or start a new one. When commanded to start the counting process, all five channels are active and count over the same counting interval. At the end of a counting cycle, the contents of all five counters and the time interval are stored in the instrument's data file, along with the date and time.

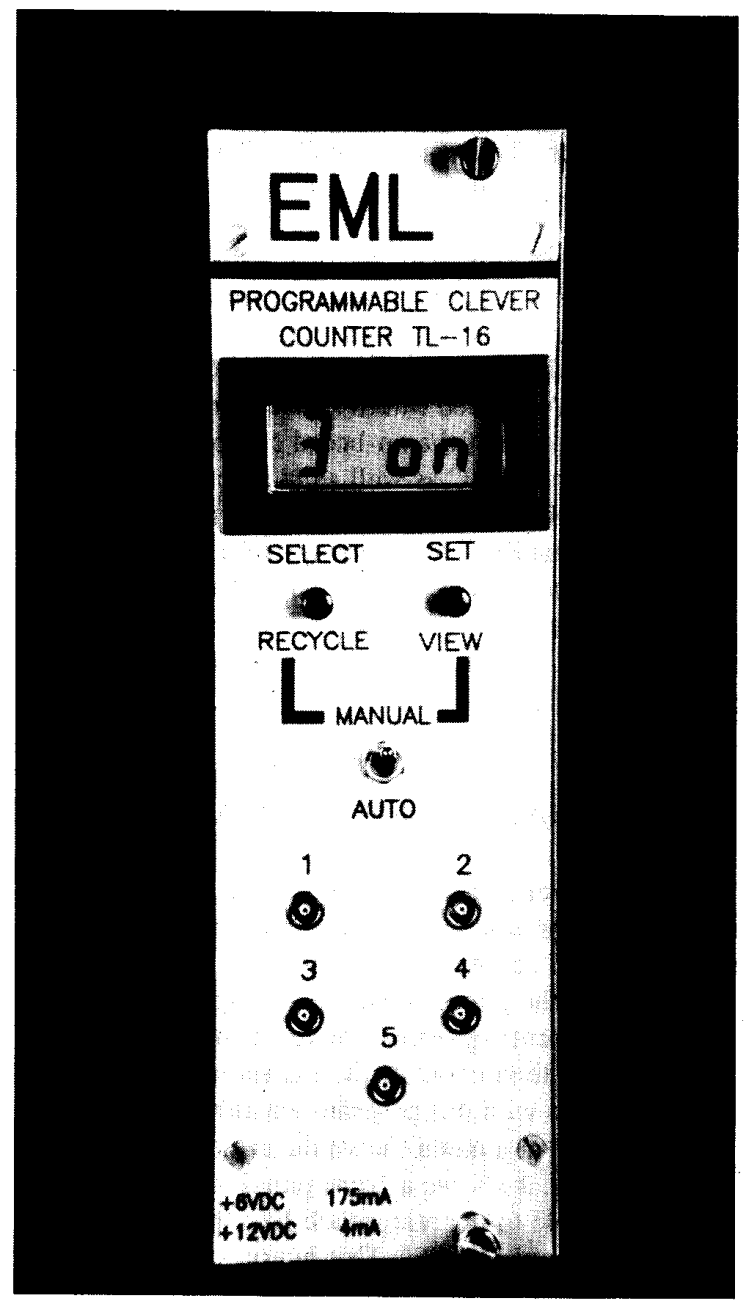

Fig. 1. The five-channel programmable counter.
A liquid-crystal display (LCD) on the front panel shows the count values as they are accumulated, as well as final results at the end of a counting cycle. Although all five channels are always active, only those chosen channels of interest are shown on the front panel display. The elapsed time is also shown.

Data can be printed while accumulated or retrieved at a later time by connecting a computer, terminal or modem to the instrument's serial port. A standard, D-type, RS-232 connector is on the rear panel. Downloading the data file can be done via either ASCII format or according to xmodem protocol. The downloading process does not clear the data file; it remains intact. If the data file fills to capacity, subsequent information is then stored at the beginning of the data file, overwriting earlier data. Changing configurations of the instrument or downloading data can be done locally or remotely even while counting continues.

A switch on the front panel selects the mode of operation. In the Auto position, the counters automatically begin a new cycle when counting is completed. In the Manual position, the counters stop at the end of the time interval and hold the results for viewing until recycled manually. Viewing and recycling are controlled by the same push buttons; i.e, the push buttons serve a dual function. In either Manual or Auto modes, all results are stored in the internal data file, which has a capacity of about 500 records. This is sufficient to allow 20 days of unattended operation assuming the user's setup calls for hourly data storage.

Applications that require more than five counting channels may be met by utilizing additional units and configuring the system to readout to a single printer or external computer through a standard communications $\mathrm{T}$-switch or one of the intelligent multiport controllers available commercially. Such a controller is able to multiplex one host computer to many instruments.

Thus, the instrument meets a variety of needs in the typical counting facility, whether for short-term manual counting or for long-term unattended data logging. Multiple counting channels can serve to simultaneously count independent samples, such as in a routine alpha or beta monitoring facility. Multiple counting channels can also permit correlated data to be accumulated when concurrent events, such as coincident alpha and beta activity, are of interest.

\section{SYSTEM DESIGN}

\section{A. Hardware}

A simple, single-board computer approach to the design of a counter results in an instrument which, while inherently flexible, counts far too slowly to be useful in most counting applications. This is because of the long processing time required to service each count. Implementing multiple counting channels on such a device is virtually impossible for any practical applications. Discrete logic devices, on the 
other hand, are capable of high counting rates but are inherently complex and inflexible. A further limitation is their small counting capacity, usually one or two binary coded decimal (BCD) digits or at most a few decades. The design described here combines the inherent advantages of both of these devices. The VLSI (Am9513A) device handles the high counting rates (up to $7 \mathrm{MHz}$ ), while the single-card computer takes care of the control and allows virtually unlimited counting capacity. The method used to accomplish this was to interface the VSLI device to the address and data busses of the single-card computer. A bidirectional buffer handles data-bus flow. It is controlled by the decoder, which translates signals from the address bus so as to determine the direction of data flow to and from the VSLI device. (These signals control the functions of the Am9513A.) This allows the assembly language program in the on-board computer to access the internal registers of the Am9513A that configures it as five independent counters. These routines also control (start, stop, reset) the counters and allow readout of their contents. Fig. 2 shows a block diagram of this overall scheme.

Fig. 3 shows a photograph of the circuit boards. The unfilled socket is for a ribbon cable to the front panel display. The small size of this circuit $(9.5 \times 6 \mathrm{~cm})$ is not only due to using a VSLI circuit, but is also a result of the dense routing ability of computer-aided design for layout of the printed circuit board. This board was designed with the aid of PCAD software from Personal CAD Systems, Incorporated. The physical layout allows the circuit board to fit over the on-board computer and mate with its connectors.
The memory circuit of the computer is first removed from its socket and placed on the circuit board; thus, affording a simple and direct means of connecting to the data and address busses.

The on-board computer, shown in Fig. 2, has extensive input/output (I/O) capability. The front panel controls and LCD use this capability extensively for examining or changing the setup and for displaying counting information as it is accumulated. Sufficient $\mathrm{I} / \mathrm{O}$ capability remains to allow the user to synchronize the counting process with the actions of external devices, such as automatic sample changers, pumps, etc. This feature is not included in our design since it was not needed for our applications. However, it can easily be implemented by merely installing additional connectors to the computer's I/O lines and making minor modifications to the software.

The on-board computer card handles the RS-232 serial communications port. It also has analog measurement capability. Adding analog measurements, such as temperature, can also be done easily, although no connectors are presently provided. Simple modifications to the instrument to allow inputs to the on-board computer's 10 analog-todigital converter (ADC) channels could allow the user to integrate simultaneous analog measurements into his/her counting applications. Some additional BASIC programming is all that would be required.

All integrated circuits, except the Am9513A, are low power complementary symmetry metal oxide semiconductor (CMOS) devices. Current drain from the $+6 \mathrm{~V} \mathrm{DC} \mathrm{NIM}$ supply is $175 \mathrm{~mA}$. Four milliamps from the $+12 \mathrm{~V} \mathrm{DC}$

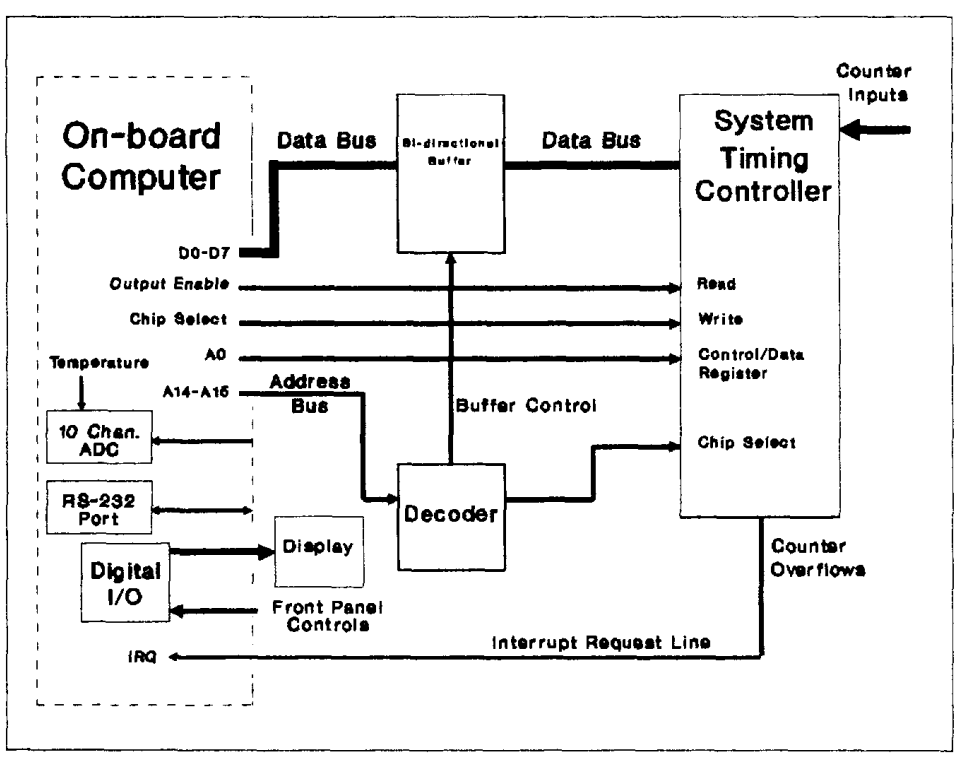

Fig. 2. Block diagram of programmable counter. 


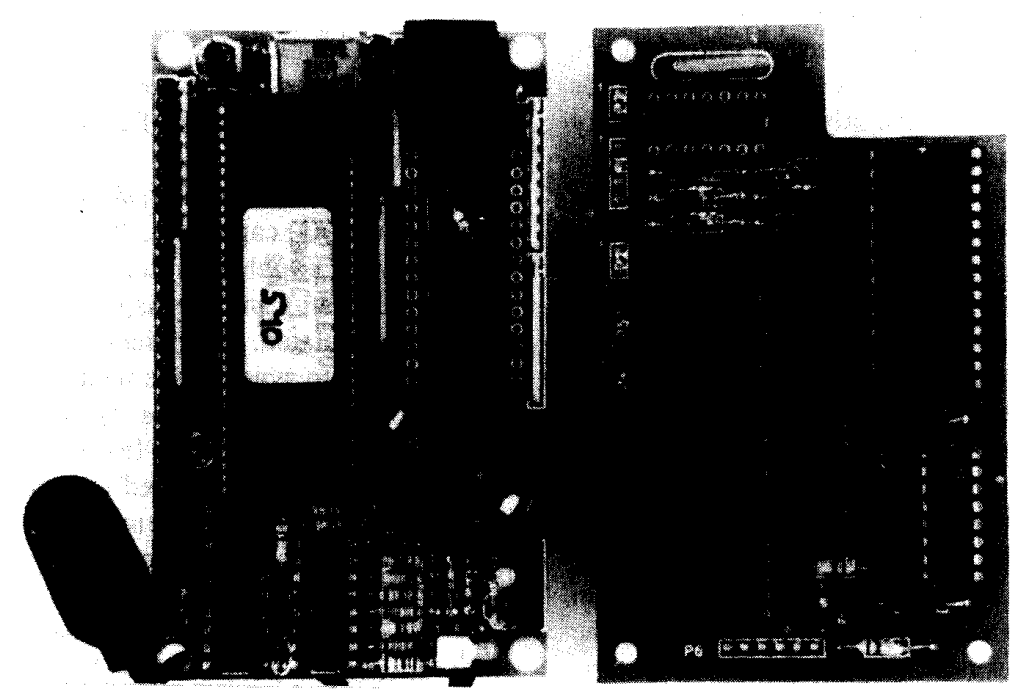

Fig. 3. The programmable counter circuit board (right) and single-card computer (left).

supply drive the $\mathrm{RS}-232$ port. Input pulses to the instrument should be +1.8 to $+5 \mathrm{~V}$ in amplitude. Circuit diagrams and additional information can be found in an EML report [5].

\section{B. Software}

The Am9513A is configured as five general-purpose, 16-bit counters. Since the capacity of each counter is limited to $65 \mathrm{k}$, more capacity is added in the on-board computer by adding an additional byte in RAM to each counter. This extends the overall capacity of each counter to over one million counts. As shown in Fig. 2, counter overflows are connected directly to the microprocessor's interrupt request (IRQ) line. When a counter overflows, an assembly language routine polls the five counters to determine which counter overflowed and updates its associated high-order byte in RAM. Additional counting capacity is easily added, if necessary. A major benefit of this technique is that counting takes place transparent to the execution of the menu-driven BASIC program.

Information from the counters is stored directly in BASIC array variables. This facilitates their manipulation. The user who desires to modify the current program need only refer to these variables to obtain count values. This could be done, for example, to customize printout or storage formats or to perform calculations based on count values. By making various calls to certain other BASIC variables, the user can also invoke the necessary assembly routines to control the counters. The user can even delete the current operating program and write his/her own program. The BASIC calls to these variables retain control over the counters. A feature of the on-board computer is that even if power should fail, a back-up battery preserves the program, the data file and all variables.

\section{SUMMARY}

A versatile and flexible five-channel counting system with on-board data storage has been developed. These twowide NIM systems have ample room for expansion, such as adding signal conditioners for analog signals, discriminators for digital signals and voltage to frequency converters (VFCs) for situations that require signal averaging. A number of these instruments are currently successfully being used in our Laboratory in various counting applications.

\section{REFERENCES}

[1] Standard Nuclear Instrumentation Modules, TID20893 (Rev. 4), July 1974. (Available from Superintendent of Documents, U.S. Government Printing Office, Washington, D.C. 20402).

[2] Polito, M. D. and V. C. Negro, "SMART-I and Clever Counter, Environmental Data Acquisition Systems," IEEE Transactions on Nuclear Science, vol. NS-26, No. 1, February 1979.

[3] Tattletale Single-Card Data Loggers, October 1989. Onset Computer Corp., P.O. Box 1030, 199 Main Street, N. Falmouth, MA 02556-1030.

[4] Am9513A/Am9513 System Timing Controller Technical Manual, Advanced Micro Devices, Inc., 901 Thompson Place, P.O. Box 453, Sunnyvale, CA 94086.

[5] Polito, M. D., "The EML TL-16, Programmable Clever Counter", EML Technical Report, in press. 